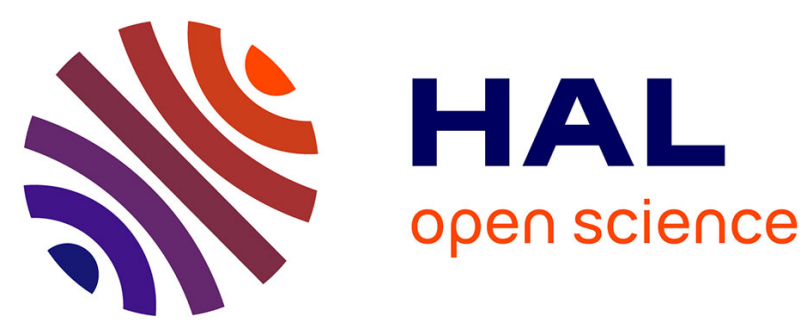

\title{
Direct amplification of ultrashort pulses in $\mu$-pulling-down Yb:YAG single crystal fibers
}

Yoann Zaouter, Igor Martial, Nicolas Aubry, Julien Didierjean, Clemens Hönninger, Eric Mottay, Frédéric Druon, Patrick Georges

\section{- To cite this version:}

Yoann Zaouter, Igor Martial, Nicolas Aubry, Julien Didierjean, Clemens Hönninger, et al.. Direct amplification of ultrashort pulses in $\mu$-pulling-down Yb:YAG single crystal fibers. Optics Letters, 2011, 36 (5), pp.748. hal-00812597

HAL Id: hal-00812597

https://hal-iogs.archives-ouvertes.fr/hal-00812597

Submitted on 15 Apr 2013

HAL is a multi-disciplinary open access archive for the deposit and dissemination of scientific research documents, whether they are published or not. The documents may come from teaching and research institutions in France or abroad, or from public or private research centers.
L'archive ouverte pluridisciplinaire HAL, est destinée au dépôt et à la diffusion de documents scientifiques de niveau recherche, publiés ou non, émanant des établissements d'enseignement et de recherche français ou étrangers, des laboratoires publics ou privés. 


\title{
Direct amplification of ultrashort pulses in $\mu$-pulling-down Yb:YAG single crystal fibers
}

\author{
Yoann Zaouter ${ }^{1, *}$ Igor Martial, ${ }^{2,3}$ Nicolas Aubry, ${ }^{3}$ Julien Didierjean, ${ }^{3}$ Clemens Hönninger, ${ }^{1}$ \\ Eric Mottay, ${ }^{1}$ Frederic Druon, ${ }^{2}$ Patrick Georges, ${ }^{2}$ and François Balembois ${ }^{2}$ \\ ${ }^{1}$ Amplitude Systemes, 6 allée du Doyen Georges Brus, 33600, Pessac, France \\ ${ }^{2}$ Laboratoire Charles Fabry de l'Institut d'Optique, CNRS, Université Paris-Sud, \\ RD 128 Campus Polytechnique, 91127, Palaiseau Cedex, France \\ ${ }^{3}$ Fibercryst SAS, La Doua-Bâtiment l'Atrium, Boulevard Latarjet, F- 69616 Villeurbanne Cedex, France \\ ${ }^{*}$ Corresponding author: yzaouter@amplitude-systemes.com
}

Received December 7, 2010; accepted January 6, 2011;

posted February 3, 2011 (Doc. ID 139335); published February 28, 2011

\begin{abstract}
We demonstrated that Yb:YAG single crystal fibers have a strong potential for the amplification of femtosecond pulses. Seeded by $230 \mathrm{fs}$ pulses with an average power of $400 \mathrm{~mW}$ at $30 \mathrm{MHz}$ delivered by a passively mode-locked Yb:KYW oscillator, the system produced $330 \mathrm{fs}$ pulses with an average power of $12 \mathrm{~W}$. This is the shortest pulse duration ever produced by an Yb:YAG amplifier. The gain in the single crystal fiber reached a value as high as 30 in a simple double-pass configuration. () 2011 Optical Society of America

OCIS codes: $140.3615,140.3280,140.3380,060.2290$.
\end{abstract}

Among all commercially available ytterbium-doped crystals, Yb:YAG has been the most intensively used in the past 20 years. This material possesses a good thermal conductivity $\kappa \sim 9 \mathrm{~W} \cdot \mathrm{m}^{-1} \cdot \mathrm{K}^{-1}$ (for a $2 \%$ doping concentration) and an unmatched emission cross section of $\sigma_{\text {emission }}=2.10^{-20} \mathrm{~cm}^{2}$ compared to most other $\mathrm{Yb}$-doped materials. Those properties make it particularly interesting for high-power systems [1]]. Furthermore, its gain bandwidth of $\Delta \lambda \sim 9 \mathrm{~nm}$, despite being among the narrowest of the $\mathrm{Yb}$-doped matrices, is commonly used for picosecond pulse amplification and still supports the production of femtosecond pulses. In the context of subpicosecond pulses, it is worth mentioning the performance obtained with thin disk Yb:YAG oscillators, with an average power above $70 \mathrm{~W}$ and a pulse energy in the multi-microjoule level [2]. The pulse duration for this kind of device, however, remains close to $1 \mathrm{ps}$. Pulsed amplifiers have also been developed with Yb:YAG, the most impressive being the Innoslabs performance with $400 \mathrm{~W}$ of average power, a pulse duration of $682 \mathrm{fs}$, and an energy in the microjoule range [B] disks and Innoslab suffer from their inherent complexity: the number of passes for the pump or for the signal is important. Amplification in Yb:YAG single crystal fibers (SCF) appears as an emerging alternative strategy for ultrashort pulse amplification to high average power in a simple setup. Indeed, an SCF possesses the aspect ration of a short rod fiber or a thin and long crystal [四]. Therefore, such an SCF can benefit from both the advantages of fibers, straightforward thermal management, and the spectroscopic and thermomechanical properties of $\mathrm{Yb}$ :YAG. However, to benefit from a high gain from this architecture, the diameter of the SCF should be $1 \mathrm{~mm}$ or below what is hardly obtainable by Czochralski or ceramic techniques. Fortunately, the micro-pulling-down crystal growth is now a mature technique remarkably suited for the production of long (up to $1 \mathrm{~m}$ ) and thin (down to $300 \mu \mathrm{m}$ diameter) Yb:YAG SCFs with laser optical quality [5]. Interesting laser performances have been reported in $\mathrm{cw}$ and $Q$-switched Yb:YAG SCF oscillators [6].
However, Yb:YAG SCFs were neither used as an amplifier nor with femtosecond pulses.

In this Letter, we report on the first (to our knowledge) demonstration of a micro-pulling-down ytterbium-doped YAG SCF ultrashort pulse amplifier.

The experimental setup is represented in Fig. 1 1 . The femtosecond pulses comes from a passively mode-locked $\mathrm{Yb}^{3+}: \mathrm{KYW}$ oscillator delivering a stable train of ultrashort pulses at a repetition rate of $30 \mathrm{MHz}$ with an average power of $600 \mathrm{~mW}$ and a transform-limited pulse duration of $220 \mathrm{fs}$. After being optically isolated, a half-wave plate and a polarizer were used to vary the signal power incident to the SCF. These pulses were directly seeded into the $1 \mathrm{~mm}$-diameter, $40 \mathrm{~mm}$-long, and 1\%-doped Yb:YAG SCF (provided by FiberCryst) amplifier by means of a $300 \mathrm{~mm}$ focal length lens. Because of the losses of the different optical components, the average power incident on the SCF was reduced to $400 \mathrm{~mW}$ at maximum. The beam waist was located at the middle of the gain medium and had a diameter of $320 \mu \mathrm{m}$. The SCF was mounted in a water-cooled copper heat sink with a temperature regulated at $10^{\circ} \mathrm{C}$. Both ends of the Yb:YAG SCF were polished at $0^{\circ}$ and antireflection coated for both pump and laser wavelengths. The gain medium was end pumped by a $200 \mu \mathrm{m}$ fiber-coupled laser diode emitting up to $180 \mathrm{~W}$ at $940 \mathrm{~nm}$ (NA of 0.22 ). The pump light was focused inside the SCF on a $400 \mu \mathrm{m}$-diameter spot size by two doublets with $50 \mathrm{~mm}$ and $100 \mathrm{~mm}$ focal length. At first the SCF amplifier was studied in single pass for gain

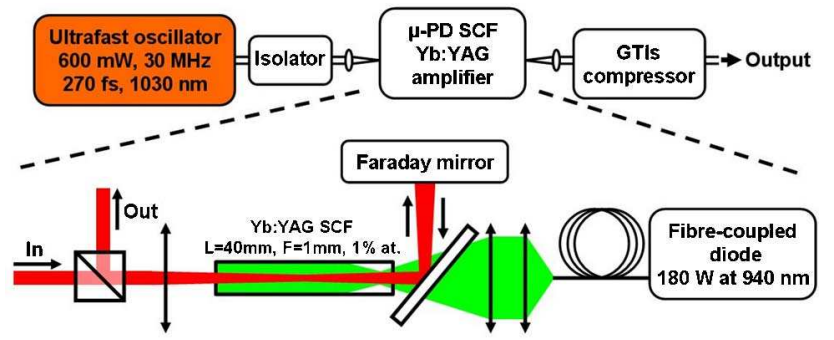

Fig. 1. (Color online) Experimental setup with a close up on the Yb:YAG SCF amplifier setup in double-pass configuration. 
characterization, pump beam and signal beam being in counter propagation. A dichroic mirror, highly transparent at $940 \mathrm{~nm}$ and highly reflective at $1030 \mathrm{~nm}$, allowed extraction of the amplified pulses. The experimental setup can also be modified for a second signal pass by the use of a Faraday mirror. In this configuration, the output after the second pass was achieved on a polarizer placed upstream the SCF amplifier. Finally, a set of GiresTournois interferometer (GTI) mirrors was implemented to compensate for the dispersion accumulated through the experiment.

In the first part of the experiment, the gain properties of the Yb:YAG SCF were studied. Figure 2(a) shows the evolution of the gain of the amplifier as a function of the input power. For the maximum input power of $400 \mathrm{~mW}$, we could extract as much as $4 \mathrm{~W}$ leading to a single-pass gain of 10. For an input average power of $1 \mathrm{~mW}$, corresponding to the lowest measurable power in our experiment, the gain reached about 21 . This means that the small signal gain is higher than this value and is remarkably high compared to other Yb:YAG amplifiers, which heightens the advantage of the SCF geometry for the realization of simple and robust amplifiers. As an example, the small signal gain obtained with similar pump power on bulk Yb:YAG was only of 3.1 []] and necessitated the use of a complex regenerative amplifier configuration for efficient amplification. Despite this high gain value, the average power of amplified spontaneous emission was not measurable at the output of the amplifier, showing that this effect was not a performance limitation with this Yb:YAG SCF.

In our setup, a simple double-pass configuration should lead to significant output power with a small signal gain of more than 400 . This was achieved in a next step as described previously on the experimental setup. Figure 2(b)] compares the output power versus the pump power in single-pass and in double-pass configuration. The curves show no sign of saturation, meaning that the thermal effects on the gain remain small, as expected in this weakly doped and long crystal. The maximum extracted average power is tripled in the second pass with respect to the first pass and reaches $12 \mathrm{~W}$ for $175 \mathrm{~W}$ of pump power with a slope efficiency of $7 \%$. The moderate efficiency obtained here may have three origins. First, with a value of 3 in the second pass, the gain is far from complete saturation and higher efficiency can be expected for higher input power. Second, the overlap between the pump and the signal is excellent in the SCF section where the pump is in free space propagation
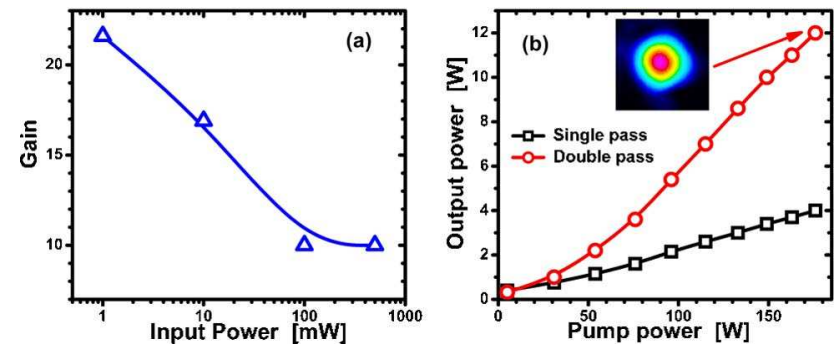

Fig. 2. (Color online) (a) Gain of the Yb:YAG SCF amplifier versus signal input power. (b) Output power after one or two passes versus pump power. Inset, spatial profile at maximum power. but becomes limited once the pump is guided by totally internal reflection. However, this moderate efficiency needs to be compared with the performance of other amplifiers: regenerative amplifiers have generally low efficiency because of the introduction of lossy components, e.g., Pockels cell. As an example, in [7], the output power was $10 \mathrm{~W}$ for $200 \mathrm{~W}$ of pump power. The most efficient $\mathrm{Yb}$ :YAG amplifier, to our best knowledge, is the Innoslab [3], with an efficiency reaching $41 \%$. But the system is used as a power amplifier, with a more powerful input signal $(2 \mathrm{~W})$ and is also more complex, with nine passes through the gain medium and a challenging reshaping of the pump beam prior to injection into the gain medium. And finally, the whole pump power is absorbed, meaning that either a shorter SCF or a lower doping concentration could prevent reabsorption effects and, thus, improve power extraction.

To finalize the characterization of the SCF amplifier, the beam profile was taken at maximum average power (after the GTI compressor). It is shown on inset of Fig. 2(b), $M^{2}$ measurements were carried out and revealed a degradation of the beam quality for increasing pump power. At $12 \mathrm{~W}$ of average output power, we measured $M_{x}^{2}=1.28$ and $M_{y}^{2}=1.68$, while the oscillator beam profile had a $M^{2}<1.2$ on both axes. This degradation is a manifestation of thermal stresses inside the gain medium. Nevertheless, we believe that this degradation could be easily limited with a better contact between the SCF and its heat sink.

In the second part of the experiment, we focused on the spectral and temporal properties of the amplified pulses after two passes in the gain medium. In order to see the effect of the gain-induced spectral narrowing, we varied the pump power of the amplifier and measured the amplified spectrum for each pump power. Figure $3(\mathrm{a})$ shows the spectral shape of the amplified pulses for different gain values. As expected, the spectrum narrowed for increasing gain. Figure 3(b) gives a more accurate idea of the evolution of the spectral bandwidth (full width at half-maximum [FWHM]) with respect to the gain of the amplifier. The pulse spectral bandwidth decreased from $5.7 \mathrm{~nm}$ (input pulses) to $3.1 \mathrm{~nm}$ at a gain of 5 and to $2.6 \mathrm{~nm}$ at a gain of 30, i.e., at the maximum extracted power of $12 \mathrm{~W}$. The latter value remains relatively high for an Yb:YAG amplifier. As an example, it was $1.81 \mathrm{~nm}$ in the Innoslab system with a gain of 200 and it was generally reduced to less than $1 \mathrm{~nm}$ in $\mathrm{Yb}: Y A G$ regenerative amplifiers where the overall gain was very high. Therefore, the
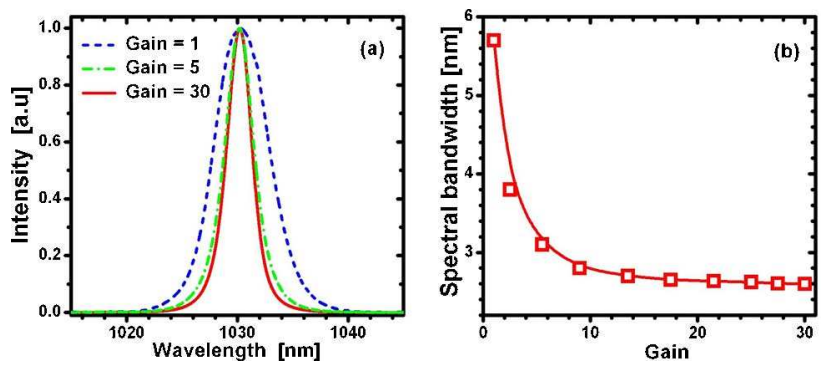

Fig. 3. (Color online) (a) Spectrum of the output pulses (after two passes) for three gain values (b) Spectral bandwidth (FWHM) versus gain of the double-pass amplifier. 

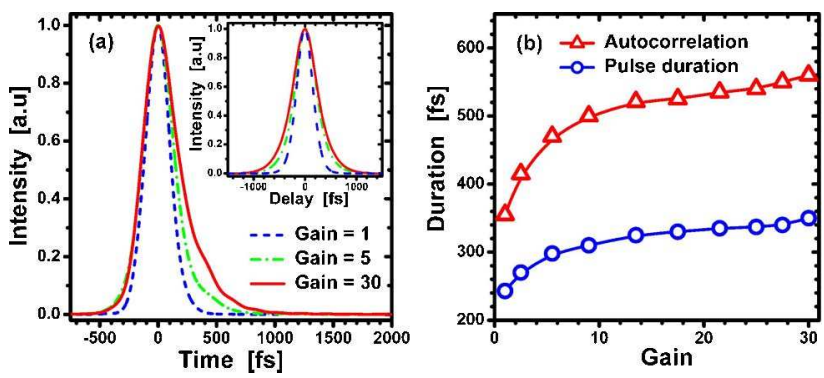

Fig. 4. (Color online) (a) Temporal profiles retrieved from FROG measurements for different amplifier gains. Inset, respective autocorrelation traces. (b) Pulse and autocorrelation FWHM durations versus amplifier gain.

gain of 30 seems to be a good compromise between significant amplification and preservation of the spectral bandwidth. Simultaneously, the pulse duration was monitored by second-harmonic-generation frequencyresolved optical gating (SHG-FROG) measurements and with an independent autocorrelator. All acquired FROG traces were retrieved with an error $<17.10^{-4}$ on $256 \times$ 256 grids. We first measured the pulse duration directly at the output of the SCF and found an autocorrelation pulse duration of $700 \mathrm{fs}$, at least 1.4 times the Fourier limit. A SHG-FROG measurement carried out highlighted an important uncompensated phase. We attribute this remaining phase to the dispersion that is added to the pulses throughout the experiment (mainly by the Faraday isolator, the Faraday mirror, and the gain medium). A GTI mirror-based compressor was, therefore, implemented to retrieve the shortest possible pulse duration. The GTI mirror compressor consisted of two types of mirrors: $-550 \mathrm{fs}^{2}$ mirrors, on which as high as 16 total bounces were made, and $-900 \mathrm{fs}^{2}$, on which the maximal total number of bounces were 8. Figure (4a) presents FROG traces (and autocorrelation traces in inset). The asymmetric temporal profile of the amplified pulses comes from a third-order dispersion-dominated remaining spectral phase that could not be fully compensated by the GTI mirror compressor. Figure [1(b) presents the pulse duration measured for different gain values provided by the amplifier. The pulse duration increased from $250 \mathrm{fs}$ to $350 \mathrm{fs}$ at maximum pump power in agreement with the observed spectral narrowing. At maximum extracted power, the duration of $350 \mathrm{fs}$ corresponds to a time-bandwidth product of 0.257 , only $8 \%$ above the calculated Fourier limit. An optimized compressor could, therefore, lead to even shorter pulses. The relatively small value of the time-bandwidth product can be attributed to the spectral reshaping occurring during amplification that transforms the initial $\mathrm{sech}^{2}$-shape spectrum into a more Lorentzian-type. To our knowledge, this represents a reduction by a factor of 2 compared to the pulse duration ever produced by a femtosecond $\mathrm{Yb}: \mathrm{YAG}$ amplifier [3].

At $12 \mathrm{~W}$ of average power, the energy per pulse was $400 \mathrm{~nJ}$ and the peak power reached $1.14 \mathrm{MW}$ after compression. It was about $570 \mathrm{~kW}$ at the output of the SCF. This value is well below the threshold of self-focusing in YAG (corresponding to $1.44 \mathrm{MW}$ ). As confirmed by the spectra showing no sign of self-phase modulation, the SCF amplifier operates below nonlinear effects even without the use of a chirped-pulse amplification scheme thanks to the large mode in the short gain medium. This represents an important advantage over fiber amplifiers as rod-type fibers [8,9] for future energy scaling.

In conclusion, we have demonstrated that Yb:YAG single crystal fibers have a strong potential for femtosecond pulse amplification. Compared to other Yb:YAG amplifiers, the main advantage of this geometry is that it provides a high single-pass gain leading to a very simple amplifier geometry, nearly as simple as conventional fiber amplifiers. Remarkably, the ultrashort pulses have been amplified up to $400 \mathrm{~nJ}$ in a chirpedpulse amplification-free configuration without manifestation of nonlinearities. Moreover, the pulse durations obtained after compression are the shortest ever obtained with an Yb:YAG amplifier, to our best knowledge. We also believe that the optical-to-optical efficiency and beam quality could be improved with more advanced SCF and cooling designs. Furthermore, the authors believe that this amplifier technology could be advantageously combined to already well-established bulk or fiber amplification schemes operating at lower repetition rates to boost their average power in the $10-100 \mathrm{~W}$ range with energies exceeding several millijoules and a pulse duration still below 500 fs.

\section{References}

1. H. Bruesselbach and D. S. Sumida, IEEE J. Sel. Top. Quantum Electron. 11, 600 (2005).

2. J. Neuhaus, D. Bauer, J. Zhang, A. Killi, J. Kleinbauer, M. Kumkar, S. Weiler, M. Guina, D. H. Sutter, and T. Dekorsy, Opt. Express 16, 20530 (2008).

3. P. Russbueldt, T. Mans, G. Rotarius, J. Weitenberg, H. D. Hoffmann, and R. Poprawe, Opt. Express 17, 12230 (2009).

4. M. J. F. Digonnet, C. J. Gaeta, and H. J. Shaw, J. Lightwave Technol. 4, 454 (1986).

5. D. Sangla, N. Aubry, J. Didierjean, D. Perrodin, F. Balembois, K. Lebbou, A. Brenier, P. Georges, O. Tillement, and J. M. Fourmigué, Appl. Phys. B 94, 203 (2009).

6. D. Sangla, I. Martial, N. Aubry, J. Didierjean, D. Perrodin, F. Balembois, K. Lebbou, A. Brenier, P. Georges, O. Tillement, and J. M. Fourmigué, Appl. Phys. B 97, 263 (2009).

7. K. Sueda, S. Kawato, and T. Kobayashi, Laser Phys. Lett. 5, 271 (2008).

8. F. Röser, T. Eidam, J. Rothhardt, O. Schmidt, D. N. Schimpf, J. Limpert, and A. Tünnermann, Opt. Lett. 32, 3495 (2007).

9. Y. Zaouter, D. N. Papadopoulos, M. Hanna, J. Boullet, L. Huang, C. Aguergaray, F. Druon, E. Mottay, P. Georges, and E. Cormier, Opt. Lett. 33, 107 (2008). 